\title{
Short Technical Report Improved Sensitivity for Solid-Support Invasive Cleavage Reactions with Flow Cytometry Analysis
}

BioTechniques 34:198-203 (January 2003)

\author{
P. Wilkins Stevens, K.V.N. \\ Rao, J.G. Hall, V. Lyamichev, \\ B.P. Neri, and D.M. Kelso \\ Northwestern University, \\ Evanston, IL, and Third Wave \\ Technologies, Madison, WI, \\ USA
}

\section{ABSTRACT}

A new configuration of the solid-support invasive cleavage reaction provides a small reaction-volume format for high-sensitivity discrimination of nucleic acid targets with single nucleotide differences. With target concentrations as low as 2 amol/assay, the solid-support invasive cleavage reaction clearly distinguishes single base mutations. Two oligonucleotides tethered to the solid support hybridize to the target nucleic acid, forming a tripartite substrate that can be recognized and cleaved by Cleavase ${ }^{\circledR}, a$ structure-specific 5'-nuclease. Each cleavage event yields fluorescence signal on the surface. When microspheres serve as the solid-support surface, analysis by fluorometer imparts real-time information about change in the reaction signal over time. Flow cytometry provides an alternative detection technology that collects endpoint information about the reaction signal on individual microspheres. A reaction volume of $10 \mu \mathrm{L}$ with as few as 3000 microspheres is sufficient to distinguish single nucleotide differences at target concentrations less than $200 \mathrm{fM}$. This sensitivity level is within the range required for analysis of SNPS in genomic DNA. In addition, the flow cytometry format has multiplexing potential, making the microsphere-based invasive cleavage assay attractive for high-throughput genomic applications.

\section{INTRODUCTION}

Capable of distinguishing nucleic acid sequences that differ at only a single nucleotide position, the invasive cleavage reaction has become a valuable tool for genomic applications, including scoring SNPs $(4,5,7,9,11,13)$, tracking genotypes of viruses and microorganisms (3), and monitoring gene expression (7). The method positions two overlapping oligonucleotides, the probe and upstream oligonucleotide, on the target nucleic acid to create a complex recognized and cleaved by a structure-specific 5' nuclease. Enzyme-substrate recognition provides the assay's exquisite discrimination of closely related nucleic acid sequences (10). Run at a temperature compatible with probe cycling, the assay exhibits signal amplification, with a single target directing cleavage of multiple-probe oligonucleotides.

Previously, we adapted the invasive cleavage reaction to a solid-support format and demonstrated effective probe cleavage whether the probe or upstream oligonucleotide or both were attached to the solid support $(8,15)$. Attachment of the oligonucleotides with long hydrophilic tethers improved reaction rates and enhanced signals (15).

Here we describe adaptations of the solid-support invasive cleavage assay that render it more suitable for diagnostic applications. As illustrated in Figure 1 , both probe and upstream oligonucleotide are bound to the solid support in this microsphere-based format. The probe is configured with a $5^{\prime}$-terminal dabcyl and an internal fluorescein; thus, microsphere-bound probe is fluorescence-quenched until target-directed cleavage releases the dabcyl quencher. After cleavage, fluorescein remains associated with the microsphere. Therefore, increased cleavage is characterized by increased microsphere fluorescence. The oligonucleotide system used in these experiments is based on the apolipoprotein E (ApoE) SNP at position 158, where $\operatorname{cgc}$ (Arg) and $\operatorname{tgc}$ (Cys) codons are represented within the population (1).

Analysis of microsphere-based invasive cleavage reactions by fluorometer yields information about the cleavage reaction in real time (15). However, sample size considerations (150-200 $\mu \mathrm{L}$ for our instrumentation) and the technique's sensitivity (approximately $500 \mathrm{fM}$ ) limit its potential for analysis of SNPs in genomic DNA. Here we demonstrate that flow cytometer analysis of the microsphere-based invasive cleavage reactions is compatible with a 20 -fold smaller reaction volume (10 $\mu \mathrm{L}$ ) and target concentrations as low as $200 \mathrm{fM}$. This high sensitivity of the solid-support invasive cleavage reaction in the flow cytometric format establishes that it is capable of distinguishing nucleic acid targets at concentrations appropriate for SNP analysis in genomic DNA. The potential for multiplexing invasive cleavage assays on tagged microspheres points to the relevance of flow cytometric analyses for future high-throughput genomic applications.

Several solution-phase SNP-genotyping methodologies have demonstrated microsphere-based formats where reaction products are captured and analyzed on coded microspheres $(2,6,14)$. The solid-support invasive cleavage reaction detailed below differs from these methodologies because the micro- 
sphere is the location where target-directed probe cleavage occurs, not just the site for capture of previously processed reaction product.

\section{MATERIALS AND METHODS}

\section{Materials}

Spherotech (Libertyville, IL, USA) donated streptavidin-coated latex microspheres and Rainbow calibration microspheres. Third Wave Technologies (Madison, WI, USA) prepared Cleavase $^{\circledR}$ enzyme as described (9). Enzyme dilution buffer, enzyme storage conditions, and oligonucleotide synthesis were specified previously (4). Phosphoramidite reagents for oligonucleotide synthesis were purchased from Glen Research (Sterling, VA, USA), Chill-out ${ }^{\mathrm{TM}} 14$ liquid wax from MJ Research (Cambridge, MA, USA), and Dbiotin from Pierce Chemical (Rockford, IL, USA). Other reagents were obtained from Sigma (St. Louis, MO, USA).

\section{ApoE System}

Sequences for Cys probe, Cys target, Arg target, and upstream oligonucleotide are in Reference 15. The Arg probe sequence is $5^{\prime}$-(dabcyl)GCTT $(F L-d T)$ TGCAGGTCATCGG $(S p)_{10}$ (biotin)-3', where $F L-d T$ is fluoresceindeoxythymidylic acid and $S p$ is the Glen Research spacer phosphoramidite 18. Each upstream oligonucleotide and probe contain 10 of these hexaethylene glycol spacers, since more effective cleavage (2- to 3-fold faster reaction rates) was observed for microspheres where both oligonucleotides were attached with linkers of the same length than when one of the two oligonucleotides was attached via a longerlength linker (data not shown).

The $\mathrm{T}_{\mathrm{m}}$ for upstream oligonucleotide-target hybridization is $83.9^{\circ} \mathrm{C}$, while $\mathrm{T}_{\mathrm{m}}$ values for probe-target hybridization are $60.0^{\circ} \mathrm{C}$ and $61.7^{\circ} \mathrm{C}$ for Cys and Arg probes, respectively. In one experiment, three upstream oligonucleotides with alternative $T_{m}$ values were tested. Sequences for these oligonucleotides are $5^{\prime}$-(biotin) $(S p)_{10}$ cactgccaggct-3'; 5'-(biotin) $(S p){ }_{10}$ GTACACTGCCAGGCT-3'; and 5'-(biotin)
$(S p)_{10}$ GGTACACTGCCAGGCT-3'. Corresponding $\mathrm{T}_{\mathrm{m}}$ values are $55.7^{\circ} \mathrm{C}$, $61.8^{\circ} \mathrm{C}$, and $65.4^{\circ} \mathrm{C}$, respectively.

\section{Preparation of ApoE Cys and Arg Microspheres}

Streptavidin-coated microspheres (diameter $3.2 \mu \mathrm{m}$, density $1.05 \mathrm{~g} / \mathrm{cm}^{3}$ ) were coated with upstream oligonu- cleotide and either Cys or Arg probe essentially as described (15), but before the coating reaction, microspheres were sonicated $30 \mathrm{~s}$ with a probe sonicator (4 W; Sonic Dismembrator 60; Fisher Scientific, Pittsburgh, PA, USA). After coating, microspheres were blocked with $10 \mu \mathrm{M}$ D-biotin. Unless otherwise stated, the ratio of probe to upstream oligonucleotide was 30:1. 


\section{Fluorometry Measurements}

For fluorometer analysis, invasive cleavage reactions were conducted at $54^{\circ} \mathrm{C}$ in $6 \times 50 \mathrm{~mm}$ disposable tubes by mixing target oligonucleotide and microspheres in a volume of $190 \mu \mathrm{L}$ reaction buffer (10 mM MOPS, pH 7.5, 7.5 $\mathrm{mM} \mathrm{MgCl}_{2}, 0.1 \%$ Tween $^{\circledR} 20,10$ $\mathrm{mg} / \mathrm{mL}$ yeast tRNA). After a $10-\mathrm{min}$ temperature equilibration at $54^{\circ} \mathrm{C}$, the reaction was initiated by addition of 10 $\mu \mathrm{L} 10 \mu \mathrm{g} / \mathrm{mL}$ Cleavase enzyme in enzyme dilution buffer. Signals generated were measured in real time, as described previously, every $15 \mathrm{~s}$ for 30-60 min with a laboratory-built fluorometer (15). Positive to negative ratios $(\mathrm{P} / \mathrm{N})$ for fluorometer data were calculated by averaging endpoint fluorometer signals for replicate reactions of Cys target with Cys microspheres (P) and dividing that average by the average of endpoint fluorometer signals for replicate reactions of Arg target with Cys microspheres $(\mathrm{N})$.

\section{Flow Cytometry Measurements}

For analysis by flow cytometer, most invasive cleavage reactions were conducted at $54^{\circ} \mathrm{C}$ in $1.7-\mathrm{mL}$ polypropylene microcentrifuge tubes by mixing target oligonucleotide, microspheres, and Cleavase enzyme in reaction buffer. Enzyme concentration was $10 \mu \mathrm{g} / \mathrm{mL}$ for reactions containing target concentrations greater than or equal to $1 \mathrm{pM}$. For lower target concentrations, the enzyme concentration was decreased to $1 \mu \mathrm{g} / \mathrm{mL}$ to reduce signal from the nonspecific target and thereby improve $\mathrm{P} / \mathrm{N}$ ratios. Reaction tubes were rocked continuously or agitated on a microplate shaker in a $54^{\circ} \mathrm{C}$ incubator for 16-20 h, unless otherwise indicated. In experiments where reaction volume was greater than $1.6 \mathrm{~mL}$, the reaction mixture was divided evenly among multiple 1.7-mL tubes. Experiments with $10-\mu \mathrm{L}$ reaction volumes, however, were conducted in $0.2-\mathrm{mL}$ MicroAmp ${ }^{\circledR}$ reaction tubes in a Perkin Elmer GeneAmp ${ }^{\circledR}$ PCR System 2400 (both from Applied Biosystems, Foster City, CA, USA).

For analysis by flow cytometry, 10$\mu \mathrm{L}$ reactions were transferred directly into $0.2 \mathrm{~mL} 0.2 \mathrm{M}$ carbonate buffer, $\mathrm{pH}$
9.4, while microspheres from reactions with larger volumes were pelleted by centrifugation at $5200 \times g$ before resuspension in $0.5 \mathrm{~mL}$ carbonate buffer and transferred to 12 × $75 \mathrm{~mm}$ tubes. Fluorescence signals were measured in logarithmic mode with a FACScan ${ }^{\mathrm{TM}}$ (BD Biosciences, San Jose, CA, USA) equipped with a $15-\mathrm{mW}$ argon-ion aircooled laser emitting 488-nm light. CELLQuest $^{\mathrm{TM}}$ Version 3.3 was the acquisition software. Rainbow calibration microspheres (Spherotech) were used as instrument calibrators, with the high voltage adjusted to approximately 825 $\mathrm{V}$ so that peak 4 read 1000 fluorescence units. Flow cytometry data were analyzed with WinMDI software, version 2.8 (http://facs.scripps.edu/software. $\mathrm{html}) . \mathrm{P} / \mathrm{N}$ values were calculated from flow cytometer data by dividing the average of median signals from replicate reactions with Cys target and Cys microspheres $(\mathrm{P})$ by the average of median signals from replicate reactions with Arg target and Cys microspheres (N).

\section{RESULTS}

\section{Solid-Support Invasive Cleavage Reactions Discriminate the ApoE Targets}

Cys microspheres were prepared with upstream oligonucleotide and Cys probe at a ratio of 30 probes/upstream oligonucleotide. Arg microspheres were prepared with upstream oligonucleotide and Arg probe at the same ratio. Fluorometer analysis of invasive cleavage reactions using Cys and Arg microspheres with each of the two targets demonstrated extensive cleavage with the complementary target but virtually no cleavage with the non-complementary target (Figure 2).

\section{$\mathbf{T}_{\mathbf{m}}$ of Upstream Oligonucleotide Affects Reaction Rate}

$\mathrm{T}_{\mathrm{m}}$ values for the upstream oligonucleotide and probe differ by more than $20^{\circ} \mathrm{C}$. Therefore, in the invasive cleav-

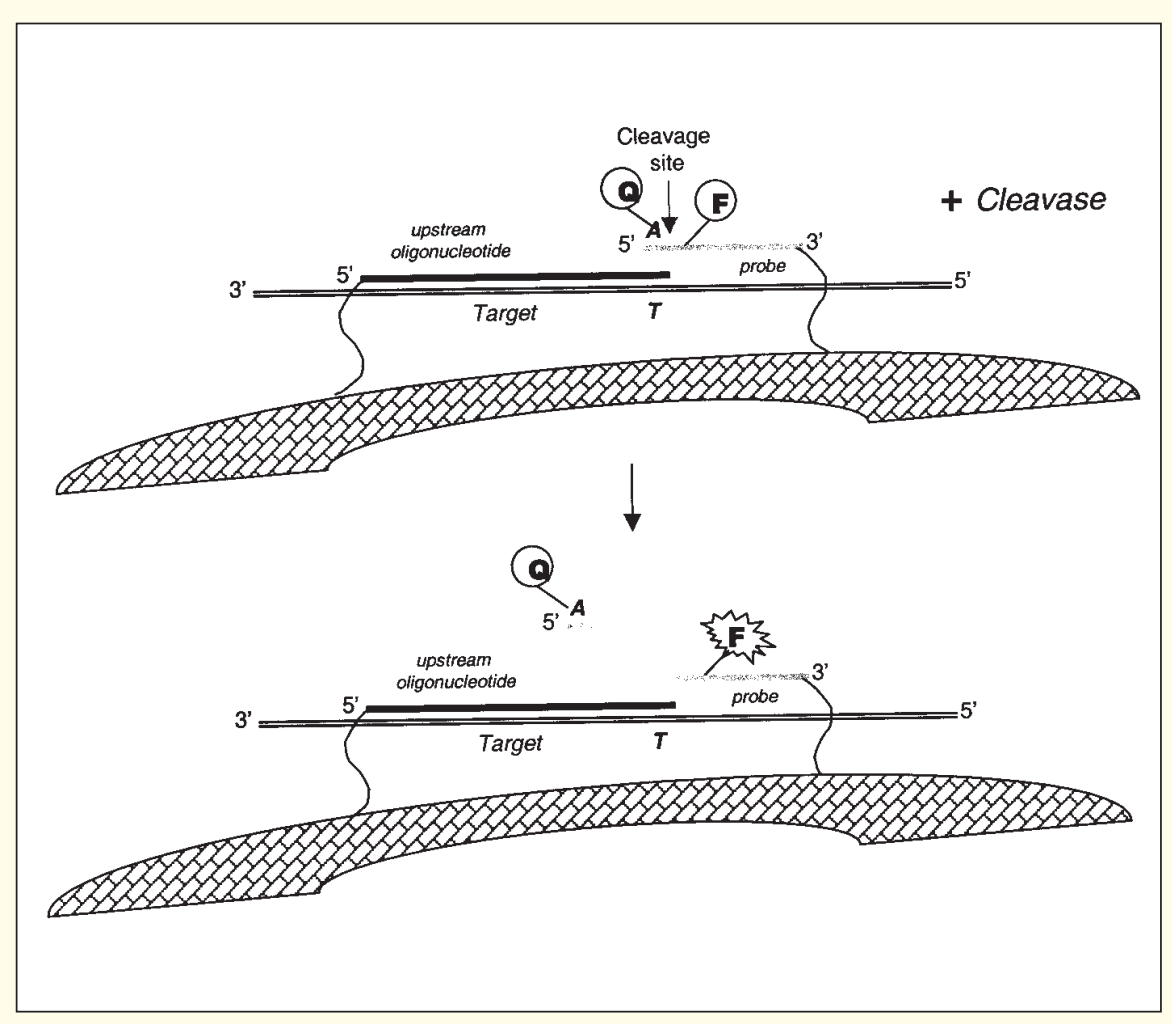

Figure 1. Schematic of solid-support invasive cleavage reaction. The probe and upstream oligonucleotide are tethered to the microsphere surface. Before cleavage, fluorescence of the fluor $(\mathrm{F})$ on the probe is quenched by the quencher moiety $(\mathrm{Q})$. The nucleotide at the target's SNP site is T. Since in the probe the base at this site is complementary to the target, the Cleavase enzyme recognizes the assembled target-probe-upstream oligonucleotide complex as a cleavable substrate. After probe cleavage and release of the quencher, the fluor on the cleaved probe exhibits fluorescence. 
age reaction, cleaved probe readily dissociates from target, while upstream oligonucleotide remains stably associated with target throughout the entire reaction. When the invasive cleavage reaction is run with all components in solution, continuous association of upstream oligonucleotide with target simplifies the assembly of the tripartite cleavable substrate. On a solid support, however, continuous association of an upstream oligonucleotide with target limits the number of probes potentially interacting with that target, since only probes in the immediate vicinity of that upstream oligonucleotide are close enough to form the required structure. Therefore, we hypothesized that allowing cycling of both probe and upstream oligonucleotide might improve cleavage rates for the solid-support reaction. To test this hypothesis, we designed three alternative upstream oligonucleotides, one with a $T_{m}$ similar to that of the probe-target $\mathrm{T}_{\mathrm{m}}$ and the other two with $\mathrm{T}_{\mathrm{m}}$ values approximately $5^{\circ} \mathrm{C}$ higher or lower than the $T_{m}$ for probe-target hybridization.

When microspheres coated with the standard Cys probe and one of the upstream oligonucleotides were reacted with Cys or Arg target, microspheres with the standard, high- $\mathrm{T}_{\mathrm{m}}$ upstream oligonucleotide performed significantly better than microspheres with any of the alternative upstream oligonucleotides. In fact, microspheres with the upstream oligonucleotide with the lowest $\mathrm{T}_{\mathrm{m}}$ demonstrated no target cleavage (data not shown).
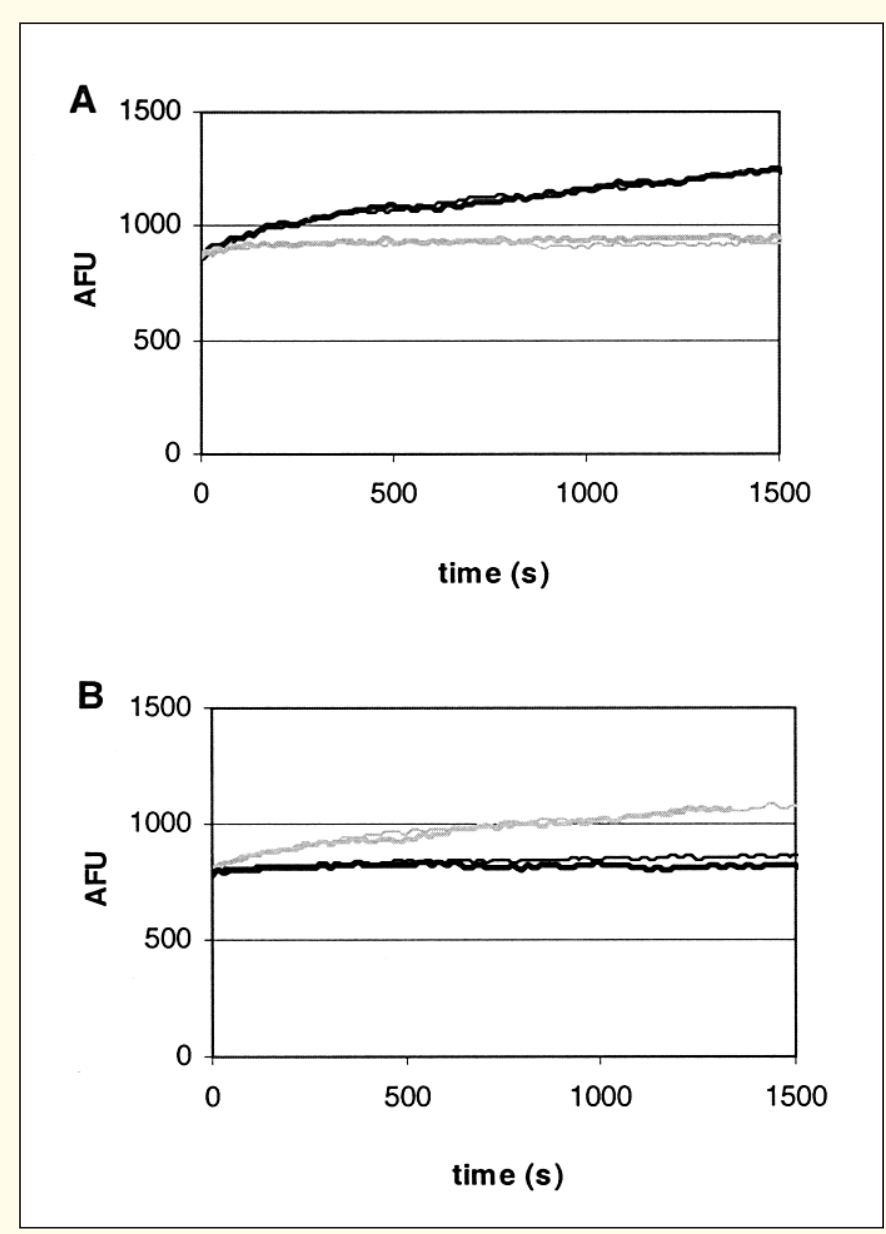

Figure 2. Real-time analysis of solid-support invasive cleavage reaction. In a $200-\mu \mathrm{L}$ reaction, $76 \mu \mathrm{g}$ Cys (A) or Arg (B) microspheres were reacted with 25 pM Cys (black) or Arg (gray) target. Reaction signal was measured every 15 $\mathrm{s}$ by fluorometer. Thick and thin lines indicate data from duplicate experiments.

Vol. 34, No. 1 (2003)

\section{Comparison of Fluorometer and Flow Cytometer Analyses}

Fluorometer measurements of solidsupport invasive cleavage record realtime signal generated by a tube of suspended microspheres and provide information about the total fluorescence of the sample's entire collection of microspheres. Flow cytometry, on the other hand, records fluorescence on a microsphere-by-microsphere basis.

To compare endpoint measurements for individual microspheres to fluorometer-based endpoint measurements, we
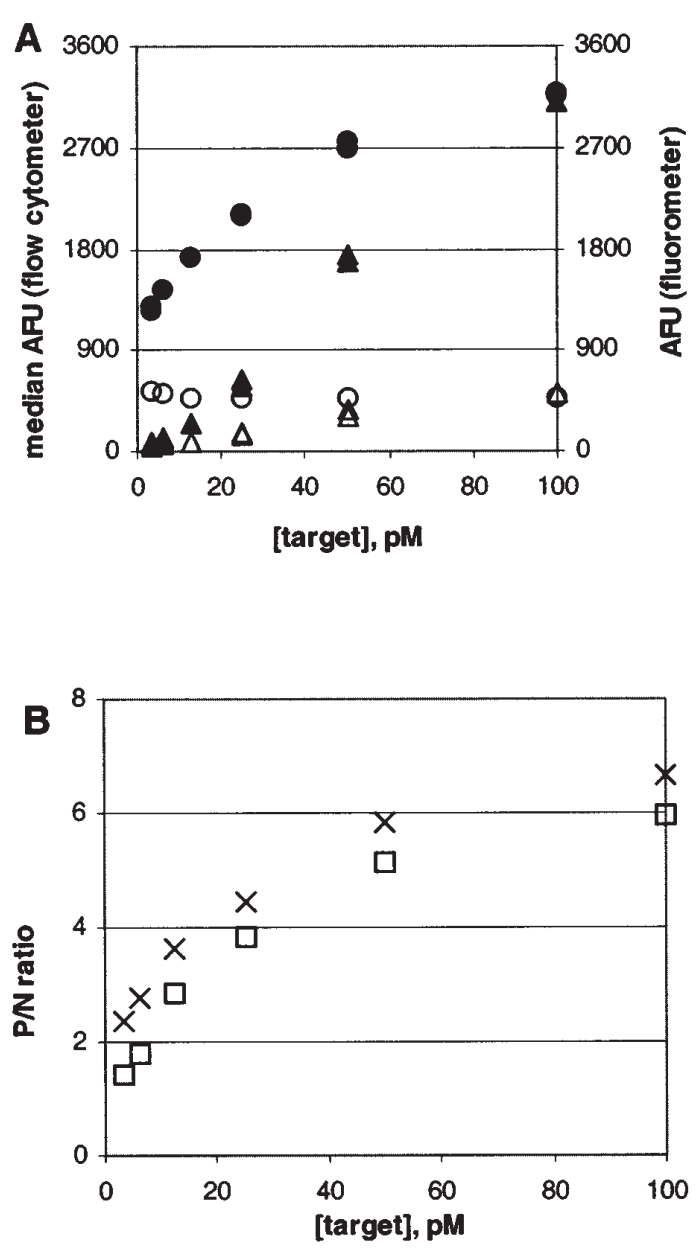

Figure 3. Comparison of reaction analysis by fluorometer versus flow cytometer measurements. (A) 2-fold dilutions of 3.2- $\mu \mathrm{m}$ Cys microspheres (from 195 to $6 \mu \mathrm{g} / \mathrm{mL}$ ) were reacted for $3 \mathrm{~h}$ with 2 -fold dilutions of Cys or Arg target (from 100 to $3 \mathrm{pM}$ ). Duplicate reactions were prepared for each concentration, and endpoint signals were measured by fluorometer (Cys target, black triangles; Arg target, open triangles). From each reaction, 10000 microspheres were read by flow cytometer, and median AFU values were determined for each Cys-target reaction (black circles) or Arg-target reaction (open circles). (B) $\mathrm{P} / \mathrm{N}$ ratios for fluorometry (open squares) and flow cytometry $(\mathrm{x})$ data shown in A. 


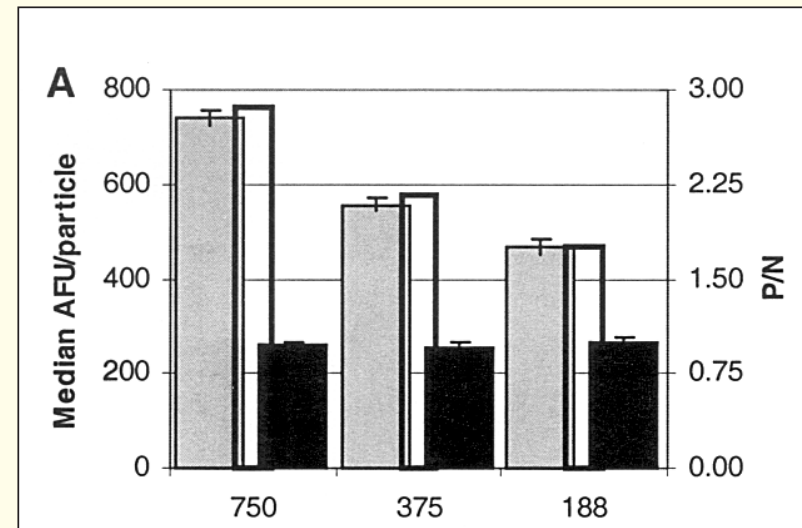

[target], $\mathrm{fM}$

B

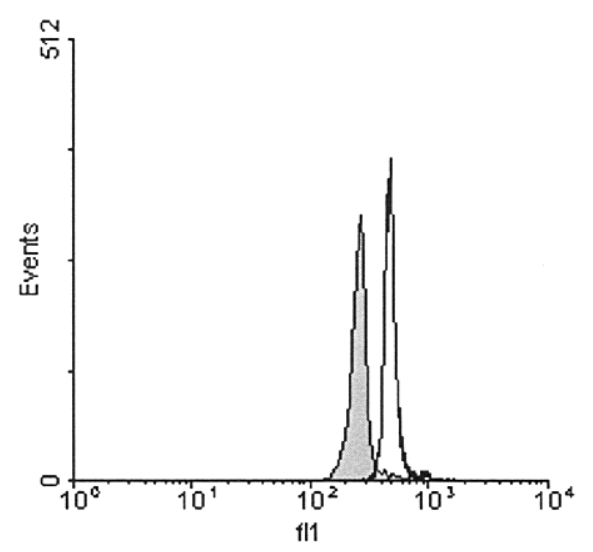

Figure 4. Reactions with low target concentrations. In a 1.6-mL reaction with $1 \mu \mathrm{g} / \mathrm{mL}$ Cleavase enzyme, $3 \mu \mathrm{g}$ (approximately 170 000) 3.2$\mu \mathrm{m}$ Cys microspheres with a 1:1 ratio of probe to upstream oligonucleotide were reacted overnight with 750,375 , or $188 \mathrm{fM}$ target. (A) Fluorescence signals from 10000 individual microspheres were measured by flow cytometer. Gray and black bars, respectively, indicate median signals for Cys-target and Arg-target reactions. Open bars represent $\mathrm{P} / \mathrm{N}$ values. (B) Flow cytometry trace for Cys microspheres reacted with $188 \mathrm{fM}$ Cys (white) or Arg (gray) target.

prepared a series of six invasive cleavage reactions containing target and microspheres at a constant ratio of 5600 target strands/microsphere. The most concentrated reactions contained $100 \mathrm{pM}$ target and $195 \mu \mathrm{g} / \mathrm{mL}$ microspheres in a reaction volume of $200 \mu \mathrm{L}$, while the most dilute reactions contained $3.1 \mathrm{pM}$ target and $6 \mu \mathrm{g} / \mathrm{mL}$ microspheres in a reaction volume of $6.4 \mathrm{~mL}$. After a 3-h incubation, fluorescence signals for each reaction were measured in the fluorometer, and then microspheres from each reaction were analyzed by flow cytometry.
Flow cytometry data showed better differentiation of microsphere signals from Cys-target and Arg-target reactions conducted with low concentrations of target and microspheres (Figure $3 \mathrm{~A}) . \mathrm{P} / \mathrm{N}$ ratios calculated from flow cytometry data followed the same trend as $\mathrm{P} / \mathrm{N}$ ratios calculated from fluorometry data, although $\mathrm{P} / \mathrm{N}$ values for flow cytometry data were consistently slightly higher (Figure 3B). These results confirmed that analysis of individual microspheres by flow cytometry was an appropriate method for analyzing solidsupport invasive cleavage reactions. In addition, increased separation of positive and negative signals and the resultant higher $\mathrm{P} / \mathrm{N}$ ratios demonstrated that flow cytometer analysis provided better sensitivity than analysis by fluorometry.

Increasing the Proportion of Upstream Oligonucleotide Improves P/N Ratios and Assay Sensitivity

Microspheres coated with Cys probe and upstream oligonucleotide at probe:upstream oligonucleotide ratios varying from $1: 1$ to $30: 1$ were tested in invasive cleavage reactions with $1 \mathrm{pM}$ target. Microspheres with equal amounts of probe and upstream oligonucleotide on the surface (1:1 ratio) demonstrated a $\mathrm{P} / \mathrm{N}$ value of 2.4. $\mathrm{P} / \mathrm{N}$ values for probe:upstream oligonucleotide ratios of $6: 1,12: 1,18: 1,24: 1$, and 30:1 were all lower: 2.2, 2.1, 1.7, 1.5 , and 1.3 , respectively. Higher proportions of probe on the microsphere surface affect $\mathrm{P} / \mathrm{N}$ ratios adversely, primarily because of the increased fluorescence of reactions with nonspecific target. High concentrations of probe contribute background fluorescence, even when microspheres have not been reacted with target or enzyme.

When microspheres coated with a 1:1 ratio of Cys probe and upstream oligonucleotide were tested in invasive cleavage reactions with Cys and Arg target, target concentrations of 750 , 375 , and $188 \mathrm{fM}$ all demonstrated significant differences in the signals generated by specific (Cys) and nonspecific (Arg) targets (Figure 4A). Even at $188 \mathrm{fM}$ target, the Cys-target and Argtarget flow cytometry peaks were well separated (Figure 4B).

\section{Solid-Support Assay Configured with Few Microspheres and Low Reaction Volume}

The solid-support invasive cleavage experiments described so far were conducted in reaction volumes of at least $200 \mu \mathrm{L}$ in microcentrifuge tubes rocked or agitated continuously to prevent microsphere settling. We wondered whether it might be possible to reduce the reaction volume and conduct solidsupport invasive cleavage reactions in a thermal cycler without any mixing.

In $10-\mu \mathrm{L}$ reaction volumes, from 3000 to 12000 microspheres coated with a 1:1 ratio of Cys probe and upstream oligonucleotide were reacted overnight with $500 \mathrm{fM}$ target at $54^{\circ} \mathrm{C}$ in a thermal cycler and then analyzed by flow cytometry. All concentrations of microspheres tested were capable of distinguishing Cys and Arg targets, as demonstrated by the $\mathrm{P} / \mathrm{N}$ ratios of 1.5 , 1.6, 1.8, and 2.1 for reactions with $12000,9000,6000$, and 3000 microspheres, respectively. The best $\mathrm{P} / \mathrm{N}$ ratio was obtained with the lowest number of microspheres.

\section{DISCUSSION}

Improving microsphere performance, decreasing microsphere number, reducing reaction volume, and analyzing reac- 
tions by flow cytometry have all contributed to significant enhancements of the solid-support invasive cleavage reaction. These enhancements resulted in overall improvement of detection sensitivity from approximately $100 \mathrm{amol}$ in a $200-\mu \mathrm{L}$ reaction $(500 \mathrm{fM})$ to $2 \mathrm{amol}$ in a $10-\mu \mathrm{L}$ reaction $(200 \mathrm{fM})$. In addition, results from $10-\mu \mathrm{L}$ reactions conducted in the thermal cycler demonstrated that microsphere-based invasive cleavage reactions can be conducted in small volumes without microsphere mixing.

The capability of doing small-volume solid-support invasive cleavage reactions means that precious samples can be conserved and that the target nucleic acid can be maintained at reasonably high concentrations. Also, reducing the number of microspheres/reaction has the potential to increase the signal generated by individual microspheres. With this small-volume system for analysis of SNPs represented at femtomolar concentrations, analysis of genomic DNA samples is feasible. With all components in solution phase, the invasive cleavage reaction is capable of SNP analysis directly from genomic DNA without up-front target amplification. The solid-support invasive cleavage assay likewise has potential for scoring SNPs directly from genomic DNA. Thus, microsphere-based reactions offer an effective platform for extending the invasive cleavage reaction from its solution-phase configuration to a solid-support format. In the future, a suspension array (12) of highly multiplexed microsphere reactions or some other array format may be useful for analysis of genomic DNAs by solidsupport invasive cleavage reactions.

\section{ACKNOWLEDGMENTS}

We thank Dr. Jeff Wang (Spherotech) for donating streptavidin-coated microspheres and flow cytometry calibration microspheres. We also thank Dr. William Miller (Northwestern University) for allowing use of the FACScan to acquire flow cytometry data.

\section{REFERENCES}

1.Breslow, J.L., J. McPherson, A.L. Nuss- baum, H.W. Williams, F. Lofquist-Kahl, S.K. Karathanasis, and V.I. Zannis. 1982. Identification and DNA sequence of a human apolipoprotein E cDNA clone. J. Biol. Chem. 257:14639-14641.

2.Cai, H., P.S. White, D. Torney, A. Deshpande, Z. Wang, B. Marrone, and J.P. Nolan. 2000. Flow cytometry-based minisequencing: a new platform for high-throughput single-nucleotide polymorphism scoring. Genomics 66:135-143.

3.Cooksey, R.C., B.P. Holloway, M.C. Oldenburg, S. Listenbee, and C.W. Miller. 2000. Evaluation of the Invader assay, a linear signal amplification method, for identification of mutations associated with resistance to rifampin and isoniazid in Mycobacterium tuberculosis. Antimicrob. Agents Chemother. 44:1296-1301.

4.Hall, J.G., P.S. Eis, S.M. Law, L.P. Reynaldo, J.R. Prudent, D.J. Marshall, H.T Allawi, A.L. Mast, et al. 2000. Sensitive detection of DNA polymorphisms by the serial invasive signal amplification reaction. Proc. Natl. Acad. Sci. USA 97:8272-8277.

5.Hessner, M.J., M.A. Budish, and K.D. Friedman. 2000. Genotyping of Factor V G1691A (Leiden) without the use of PCR by invasive cleavage of oligonucleotide probes. Clin. Chem. 46:1051-1056.

6.Iannone, M.A., J.D. Taylor, J. Chen, M.-S. Li, P. Rivers, K.A. Slentz-Kesler, and M.P. Weiner. 2000. Multiplexed single nucleotide polymorphism genotyping by oligonucleotide ligation and flow cytometry. Cytometry 39:131-140.

7.Kwiatkowski, R.W., V. Lyamichev, M. de Arruda, and B. Neri. 1999. Clinical, genetic, and pharmacogenetic applications of the Invader assay. Mol. Diagn. 4:353-364.

8.Lu, M., J.G. Hall, M.R. Shortreed, L. Wang, W.T. Berggren, P. Wilkins Stevens, D.M. Kelso, V. Lyamichev, et al. 2002. Structure-specific DNA cleavage on surfaces. J. Am. Chem. Soc. 124:7924-7931.

9.Lyamichev, V., A.L. Mast, J.G. Hall, J.R. Prudent, M.W. Kaiser, T. Takova, R.W. Kwiatkowski, T.J. Sander, et al. 1999. Polymorphism identification and quantitative detection of genomic DNA by invasive cleavage of oligonucleotide probes. Nat. Biotechnol. 17:292-296.

10.Lyamichev, V.I., M.W. Kaiser, N.E. Lyamicheva, A.V. Vologodskii, J.G. Hall, W.-P. Ma, H.T. Allawi, and B.P. Neri. 2000. Experimental and theoretical analysis of the invasive signal amplification reaction. Biochemistry 39:9523-9532.

11.Neville, M., R. Selzer, B. Aizenstein, M. Maguire, K. Hogan, R. Walton, K. Welsh, B. Neri, and M. de Arruda. 2002. Characterization of cytochrome P450 2D6 alleles using the Invader ${ }^{\mathrm{TM}}$ system. BioTechniques 32:S34S43.

12.Nolan, J.P. and L.A. Sklar. 2002. Suspension array technology: evolution of the flat-array paradigm. Trends Biotechnol. 20:9-12.

13.Olivier, M., L.-M. Chuang, M.-S. Chang, Y.-T. Chen, D. Pei, K. Ranade, A. de Witte, J. Allen, et al. 2002. High-throughput genotyping of single nucleotide polymorphisms using new biplex invader technology. Nucleic
Acids Res. 30:e53.

14.Taylor, J.D., D. Briley, Q. Nguyen, K. Long, M.A. Iannone, M.-S. Li, F. Ye, A. Afshari, et al. 2001. Flow cytometric platform for highthroughput single nucleotide polymorphism analysis. BioTechniques 30:661-669.

15.Wilkins Stevens, P., J.G. Hall, V. Lyamichev, B.P. Neri, M. Lu, L. Wang, L.M. Smith, and D.M. Kelso. 2001. Analysis of single nucleotide polymorphisms with solid phase invasive cleavage reactions. Nucleic Acids Res. 29:e77.

Received 2 July 2002; accepted 9 September 2002.

\section{Address correspondence to:}

Dr. Priscilla Wilkins Stevens

Department of Biomedical Engineering

Robert R. McCormick School of Engineering and Applied Science

Northwestern University

2145 Sheridan Road

Evanston, IL 60208-3107, USA

e-mail:pwilkins@northwestern.edu 